\title{
Autistic traits in women with primary dysmenorrhea: a case-control study
}

\author{
This article was published in the following Dove Press journal: \\ Neuropsychiatric Disease and Treatment \\ 12 September 2016 \\ Number of times this article has been viewed
}

\author{
Harun Toy' \\ Arzu Hergüner ${ }^{2}$ \\ Sevcan Șimșek' \\ Sabri Hergüner ${ }^{3}$ \\ 'Department of Obstetrics and \\ Gynecology, Meram Faculty of \\ Medicine, Necmettin Erbakan \\ University, ${ }^{2}$ Child and Adolescent \\ Psychiatry Clinic, Konya Training and \\ Research Hospital, ${ }^{3}$ Department of \\ Child and Adolescent Psychiatry, \\ Meram Faculty of Medicine, \\ Necmettin Erbakan University, \\ Konya, Turkey
}

Objectives: Recent studies have shown that women with autism spectrum disorder have higher rates of menstrual problems, including irregular menstrual cycles, unusually painful periods (dysmenorrhea), and excessive menstrual bleeding. In this study, we investigated the autistic traits in female university students with primary dysmenorrhea (PD).

Methods: Seventy females with PD and 70 females without PD were enrolled in the study. The Autism Spectrum Quotient (AQ) was used to measure autistic traits and the Brief Symptom Inventory was used for evaluating anxiety and depression levels. The dysmenorrheal pain was assessed by visual analog scale (VAS), coded from 0 to 10 . Weight and height were measured, and the body mass index was calculated.

Results: There were no statistical differences between the groups in terms of age, duration of education, and body mass index. Women with PD had higher AQ - Total, and AQ - Attention Switching subscale scores than subjects without PD. Spearman analysis revealed that AQ Total and AQ - Attention Switching scores were correlated with VAS. According to the linear regression analysis, VAS was predicted only by AQ - Attention Switching subscale.

Conclusion: Our findings showed an association between autistic traits and dysmenorrhea in typically developing females.

Keywords: primary dysmenorrhea, autistic traits, androgens

\section{Introduction}

Primary dysmenorrhea (PD) is the most prevalent gynecological condition experienced in women of reproductive age. ${ }^{1}$ It is defined as a colicky, low abdominal pain associated with menstruation in the absence of any obvious pelvic pathology. PD first occurs usually during adolescence after ovulatory cycles become established, and pain severity decreases with increasing age. ${ }^{2}$ The contributing risk factors include younger age, low and high body mass index, earlier age at menarche (AAM), smoking, prolonged or aberrant menstrual flow, family history of dysmenorrhea, nulliparity, high caffeine intake, and a history of sexual abuse. ${ }^{1-3}$ It negatively affects daily life activities, academic performance, and socializing with peers and is reported to be associated with psychiatric conditions such as depression and anxiety. ${ }^{4}$

Autism spectrum disorder (ASD) is characterized by deficits in social communication and social interaction across contexts, as well as restricted and repetitive patterns of behavior, interests, or activities. ${ }^{5}$ According to the report of Autism and Developmental Disabilities Monitoring Network, the current estimated prevalence of ASD is $14.7 / 1,000$ (one in 68) children aged 8 years. ${ }^{6}$ Males are approximately four times more likely than females to receive the diagnosis. ${ }^{7}$ Although the underlying
Correspondence: Harun Toy Department of Obstetrics and Gynecology, Meram Faculty of Medicine, Necmettin Erbakan University, S Block Fifth Floor, No 50I, Post Code 42090, Meram, Konya, Turkey

Tel +90332 2237863

Email haruntoy@hotmail.com 
etiology remains unclear, recent findings suggest that ASD is a polygenetic disorder with interaction of genetic and environmental factors. ${ }^{8}$

The androgen theory of ASD proposes that elevated prenatal androgens may have masculinizing effects on fetal brain and may be a precursor for autistic traits. ${ }^{7}$ Previous studies have shown that higher fetal testosterone levels, measured in amniotic fluid, were positively correlated with autistic traits in typically developing children. ${ }^{7,9}$ Lower second-to-fourth digit length ratio (2D:4D) was reported in individuals with ASD, indicating greater fetal testosterone exposure. ${ }^{10}$ Several studies have also demonstrated that excessive androgens have been related to menstrual problems, including amenorrhea, irregular menstrual cycles, and dysmenorrhea. ${ }^{11-13}$ The androgen theory also suggests that women with ASD might be more vulnerable to medical conditions associated with elevated androgens, including severe acne, hirsutism, and menstrual problems. ${ }^{14,15}$

Limited literature indicates that women with ASD experience higher rates of menstrual difficulties, including premenstrual syndrome, irregular menstrual cycles (amenorrhea), unusually painful periods (dysmenorrhea), and excessive menstrual bleeding. ${ }^{14-18}$ In an observer-rated study, Obaydi and Puri ${ }^{18}$ have found a markedly increased prevalence of premenstrual syndrome in a group of women with autism compared to age- and intelligence-matched controls (92\% vs 11\%). Ingudomnukul et $\mathrm{al}^{14}$ have demonstrated that significantly more women with ASD experienced menstrual problems, including irregular menstrual cycles $(57.4 \%$ vs $28.6 \%$ ) and dysmenorrhea (44.4\% vs $28 \%$ ) compared to controls. Pohl et al ${ }^{15}$ have also reported higher rates of irregular menstrual cycles $(46.3 \%$ vs $34 \%)$ and dysmenorrhea (39.3\% vs 26.3\%) among women with ASD than those among healthy controls.

ASD is a clinical diagnosis; however, behavioral and cognitive traits linked to ASD (autistic traits) are common and follow a continuous distribution in the general population. ${ }^{19,20}$ Previous studies have reported associations between autistic traits and other factors, including white matter connectivity, facial features, and genetic polymorphisms. ${ }^{21-23}$ Lundström et $\mathrm{al}^{24}$ have demonstrated that both ASD and autistic traits share etiological similarity, and they have suggested that they differ from each other in terms of severity and functional impairment.

In this study, we aimed to investigate the association between autistic traits and PD in a group of university female students. Based on the above literature, indicating that dysmenorrhea was more frequent in women with ASD than controls, we predicted that women with PD would have higher autistic traits than women without PD.

\section{Methods}

\section{Participants}

This case-control study was conducted between January 2015 and October 2015. The participants of the study were female medical students, recruited from a public university, Necmettin Erbakan University (Konya city, middle of Turkey). Menstruation pain was first measured in each individual by a visual analog scale (VAS), coded from 0 to 10 . Subjects who had a history of dysmenorrhea, starting shortly after menarche, and who rated menstrual pain as "severe", defined as a score $\geq 6$ on the VAS, ${ }^{25}$ were included in the study group (SG). Subjects were enrolled into the control group (CG) if they rated their menstrual pain as $\leq 2$ on the VAS.

Dysmenorrhea was defined as a 12-72 hours colicky pain in the lower abdomen during each menstrual cycle (for three consecutive menses), with or without nausea, diarrhea, fatigue, or headache. ${ }^{1}$ The inclusion criteria for all the participants were as follows: 1) 18- to 25-year-old females; 2) a regular menstrual cycle of $\sim 27-32$ days; and 3 ) nulliparity. The exclusion criteria were as follows: 1) acute or chronic pelvic disease; 2) physical illness causing pain; 3) smoking; 4) pregnancy or/and a history of childbirth; and 5) use of any psychotropic drugs and hormonal contraception within last 6 months.

\section{Procedure}

Before entering the study, purpose and procedure of the study were explained to the participants. Upon their agreement, written informed consent was obtained from all participants. All the subjects were clinically examined by a gynecologist and completed a comprehensive evaluation, including medical history, pelvic manual examination, and pelvic ultrasonography in the Obstetrics and Gynecology Outpatient Clinic to exclude secondary dysmenorrhea caused by organic pelvic diseases. Subjects who showed no evidence of a medical condition that would explain PD were considered eligible for the study. The participants did not use any pain-relieving medication on the day of assessment.

They were then invited to complete a data form about their demographic characteristics and menstrual patterns and to rate their current menstruation pain by using VAS. Afterward, they filled out two self-report questionnaires, Brief Symptom Inventory (BSI) for measuring their anxiety and depression levels and Autism Spectrum Quotient (AQ) to evaluate their autistic traits. Weight and height were measured in all participants, and their body mass index was calculated. This procedure was conducted in the same order and took $\sim 25-30$ minutes to complete for each subject during the menstruation phase. There was no remuneration for participation. 
The study and the study protocol were reviewed and approved by the ethics committee of the Necmettin Erbakan University, Faculty of Medicine, Konya, Turkey.

\section{Instruments}

\section{Data form}

This is a self-reported form, prepared for the purpose of this study, that included questions about the demographic characteristics (age, duration of education) and menstrual patterns (AAM, duration of the menstruation cycles and bleeding, duration of the menstruation pain) of the subjects.

\section{Menstruation pain intensity}

During their menstruation phase, the participants rated the intensity of their current menstrual pain on a $10 \mathrm{~cm}$ VAS, from "no pain" (0) to "the worst pain I have ever experienced" (10).

\section{Autism Spectrum Quotient}

The AQ is a 50-item self-report questionnaire that measures autistic traits in adults with normal intelligence.$^{20}$ Individuals are asked to indicate how well each statement applies to them on a 4-point scale: "strongly agree", "slightly agree", "slightly disagree", and "strongly disagree". Each item is rated with a score of "1" to a response indicating an autistic trait and " 0 " to a response nonindicative of an autistic trait. It consists of five subscales, each comprising ten items: Social Skills, Attention to Detail, Attention Switching, Communication, and Imagination. Items within each subscale are summed to provide a quantitative measure of that particular autistic trait, with higher scores indicating increased autistic traits. The reliability and validity of the AQ in the Turkish population was done by Köse et al. ${ }^{26}$ They reported the internal consistency as $\alpha=0.64$. In the current study, internal consistency for the AQ was $\alpha=0.55$.

\section{Brief Symptom Inventory}

The BSI is a 53-item, self-report inventory that measures symptoms of psychopathology in adults. ${ }^{27}$ The participants were asked to rate the degree to which they were affected by and experienced distress from symptoms during the previous 7 days. Each question was rated on a 5-point Likert scale ranging from "not at all" (0) to "extremely" (4). The Turkish version of BSI was reported to be reliable and valid for use in the Turkish population. ${ }^{28}$ For the current study, only subscales measuring depression and anxiety were used. In this sample, BSI - Depression and BSI - Anxiety had good internal consistency (BSI - Depression, $\alpha=0.89$; BSI - Anxiety, $\alpha=0.88)$.

\section{Data analysis}

Statistical analyses were done using SPSS for Windows, Version 22.0 (IBM Corporation, Armonk, NY, USA). Continuous variables were represented as mean \pm standard deviation. Kolmogorov-Smirnov test was performed to test distribution of the data, and according to the results, an independent $t$-test or Mann-Whitney $U$-test was used to compare continuous variables between the groups. Spearman's correlation was used to analyze the associations between VAS and study variables. Linear regression analysis was performed to determine the predictors of VAS score. A $P$-value of $<0.05$ (two tailed) was considered as statistically significant.

\section{Results}

One hundred forty female students met the criteria for participation and were enrolled into the study. They were divided into two groups based upon their VAS score: the SG $(n=70)$ had severe painful menstruation (VAS $\geq 6$ ) and the $C G(n=70)$ had no pain during menstruation (VAS $\leq 2)$. Statistical tests confirmed that the two groups were well matched for age, education, anthropometric variables, and menstrual characteristics (Table 1).

According to the VAS, women in the SG reported significantly more severe menstrual pain compared to controls (7.44 \pm 1.14 vs $0.99 \pm 0.81 ; P<0.000$ ). The menstrual pain duration of the subjects in the SG was $2.23 \pm 0.94$ days and their history of dysmenorrhea was $7.77 \pm 2.80$ years.

Table I Characteristics of the participants by groups

\begin{tabular}{|c|c|c|c|c|c|}
\hline Characteristic & SG & $\mathbf{C G}^{\#}$ & t/z score & $P$-value & $r$ \\
\hline Age (years) ${ }^{\mathrm{a}}$ & $22.13 \pm 2.01$ & $22.06 \pm 2.09$ & -0.027 & 0.978 & -0.002 \\
\hline Education (years) ${ }^{a}$ & $16.36 \pm 1.89$ & $16.14 \pm 1.75$ & -0.869 & 0.385 & -0.073 \\
\hline BMI $\left(\mathrm{kg} / \mathrm{m}^{2}\right)^{\mathrm{b}}$ & $21.25 \pm 2.34$ & $21.17 \pm 3.61$ & 0.171 & 0.865 & 0.014 \\
\hline AAM (years) ${ }^{a}$ & $12.80 \pm 1.25$ & $|3.0| \pm 1.39$ & -1.046 & 0.296 & -0.088 \\
\hline Menstrual cycle duration (days) ${ }^{\mathrm{a}}$ & $29.07 \pm 3.49$ & $28.4 I \pm 4.17$ & -1.439 & 0.150 & -0.121 \\
\hline Menstruation bleeding duration (days) ${ }^{\mathrm{a}}$ & $6.70 \pm 1.23$ & $6.56 \pm 1.35$ & -1.228 & 0.219 & -0.103 \\
\hline Menstrual pain intensity $(\mathrm{VAS}, \mathrm{cm})^{\mathrm{a}}$ & $7.44 \pm 1.14$ & $0.99 \pm 0.81$ & -10.903 & 0.000 & -0.921 \\
\hline
\end{tabular}

Notes: aMann-Whitney $U$-test. 'Independent $t$-test. "Data presented as mean \pm SD. Bold data, $P<0.05$ (significance).

Abbreviations: SG, study group; CG, control group; BMI, body mass index; AAM, age at menarche; VAS, visual analogue scale; SD, standard deviation. 
Table 2 Mean \pm SD scores of the AQ and BSI subscales by groups

\begin{tabular}{|c|c|c|c|c|c|}
\hline Subscale & SG & CG & t/z score & $P$-value & $r$ \\
\hline$A Q-$ Total $^{a}$ & $19.94 \pm 4.85$ & $18.27 \pm 4.65$ & 2.082 & 0.039 & 0.174 \\
\hline AQ - Social Skills ${ }^{b}$ & $3.77 \pm 1.93$ & $3.53 \pm 1.68$ & -0.702 & 0.483 & -0.059 \\
\hline $\mathrm{AQ}$ - Attention to Detail ${ }^{2}$ & $5.2 \mathrm{I} \pm 2.0 \mathrm{I}$ & $4.76 \pm 1.77$ & 1.426 & 0.156 & 0.120 \\
\hline $\mathrm{AQ}$ - Attention Switching ${ }^{\mathrm{a}}$ & $5.29 \pm 1.55$ & $4.7 I \pm I .69$ & 2.080 & 0.039 & 0.17 \\
\hline $\mathrm{AQ}-$ Communication $^{\mathrm{b}}$ & $2.39 \pm 2.15$ & $2.20 \pm 1.76$ & -0.083 & 0.934 & -0.007 \\
\hline $\mathrm{AQ}$ - Imagination ${ }^{\mathrm{b}}$ & $3.29 \pm 1.73$ & $3.07 \pm 1.58$ & -0.561 & 0.575 & -0.047 \\
\hline BSI - Depression ${ }^{\mathrm{a}}$ & $16.93 \pm 9.34$ & $15.50 \pm 6.79$ & 1.035 & 0.302 & 0.08 \\
\hline BSI - Anxiety ${ }^{a}$ & $13.54 \pm 8.56$ & $12.76 \pm 7.19$ & 0.588 & 0.557 & 0.04 \\
\hline
\end{tabular}

Notes: andependent $t$-test. 'Mann-Whitney $U$-test. Bold data, $P<0.05$ (significance).

Abbreviations: SD, standard deviation; AQ, Autism Spectrum Quotient; BSI, Brief Symptom Inventory; SG, study group; CG, control group.

The AQ - Total revealed that SG had higher autistic scores than controls. The mean scores in all subscales of AQ were higher in the SG than CG; however, only Attention Switching subscale reached significance. There were no significant differences between groups in terms of depression and anxiety levels (Table 2).

We examined the association between VAS and each subscale of the AQ, BSI - Anxiety, and BSI - Depression. Spearman analysis showed that VAS score was positively correlated with only AQ - Total $(r=0.190, P=0.025)$ and AQ Attention Switching subscale ( $r=0.205, P=0.015)$. Multiple linear regression analysis was performed to determine the factors affecting menstrual pain intensity, measured by VAS. Independent variables that entered into the regression analysis included AQ subscales, BSI - Depression, BSI Anxiety scores, and clinical characteristics included age, education, AAM, and duration of the menstruation cycle. $\mathrm{AQ}-$ Attention Switching subscale was found as a significant factor (Table 3).

Table 3 Linear regression analysis

\begin{tabular}{lllll}
\hline Variables & B & Beta & $\begin{array}{l}\text { 95\% confidence } \\
\text { interval }\end{array}$ & P-value \\
\hline AQ - Social Skills & 0.099 & 0.053 & $-0.290-0.487$ & 0.615 \\
AQ - Attention to Detail & 0.232 & 0.160 & $-0.085-0.548$ & 0.150 \\
AQ - Attention Switching & 0.410 & 0.198 & $0.034-0.785$ & $\mathbf{0 . 0 3 3}$ \\
AQ - Communication & -0.037 & -0.021 & $-0.382-0.309$ & 0.834 \\
AQ - Imagination & 0.226 & 0.11 I & $-0.151-0.604$ & 0.237 \\
BSI - Depression & 0.074 & 0.180 & $-0.052-0.200$ & 0.246 \\
BSI - Anxiety & -0.070 & -0.164 & $-0.203-0.062$ & 0.295 \\
Age & -0.095 & -0.058 & $-0.691-0.501$ & 0.754 \\
Education & 0.073 & 0.039 & $-0.595-0.74 I$ & 0.829 \\
BMI & -0.042 & -0.037 & $-0.24 I-0.158$ & $0.68 I$ \\
AAM & -0.081 & -0.031 & $-0.545-0.383$ & 0.729 \\
Menstrual cycle duration & 0.075 & 0.085 & $-0.078-0.227$ & 0.336 \\
\hline
\end{tabular}

Notes: $R=0.295 . R^{2}=0.087$. Bold data, $P<0.05$ (significance).

Abbreviations: AQ, Autism Spectrum Quotient; BSI, Brief Symptom Inventory; BMI, body mass index; AAM, age at menarche.

\section{Discussion}

In this case-control study, we investigated the relationship between PD and autistic traits in female university students. According to our knowledge, this is the first study examining autistic features in young females with PD by using the AQ, an established measure to screen autistic traits in the general population. We found that subjects with PD reported more autistic traits than age-matched healthy controls and AQ Attention Switching subscale was associated with menstruation pain intensity, as measured by VAS.

Our results are in line with findings of two prior studies indicating an association between dysmenorrhea and ASD. ${ }^{14,15}$ Ingudomnukul et al have examined a number of medical conditions and behavioral traits, seen as associated with elevated androgen levels, in women with ASD using an electronically distrubuted questionnaire, The Testosterone Medical Questionnaire. Several medical conditions, including polycystic ovary syndrome, hirsutism, severe acne, and menstrual problems were found at a higher rate in the ASD group $(n=54)$ than healthy controls $(n=183)$. In the study of Ingudomnukul et al, ${ }^{14}$ significantly more women with ASD reported dysmenorrhea ( $44.4 \%$ vs $28 \%$ ). Pohl et a ${ }^{15}$ replicated these findings in a larger sample and similarly reported that dysmenorrhea was more frequent in women with ASD $(n=415)$ than controls $(n=415 ; 39.3 \%$ vs $26.3 \%$ ). They suggested elevated androgen levels as the common link between the following two conditions: ASD and dysmenorrhea. ${ }^{14,15}$

Several studies have suggested that excessive androgens were related to menstrual problems, including amenorrhea, irregular menstrual cycles, and dysmenorrhea. ${ }^{11-13}$ Association between increased androgens and menstrual irregularities has been reported in both clinical and nonclinical samples. ${ }^{13,29}$ Van Anders and Watson ${ }^{13}$ have found significant positive correlations between salivary testosterone levels and menstrual irregularities in healthy women. Hart et $\mathrm{al}^{12}$ have 
demonstrated that fetal androgen exposure had an influence on reproductive function of female offspring. Our finding, higher autistic traits in women with $\mathrm{PD}$, raises the possibility that a common biological mechanism, elevated androgens, underlies both conditions.

Existing literature indicates that autistic traits exist along a continuum in the general population. The androgen theory suggests that certain groups within the general population may be predisposed to have higher autistic traits. ${ }^{7}$ A previous study showed that women with polycystic ovary syndrome, which is associated with menstrual irregularities, had higher autistic traits than controls. ${ }^{30}$ Recently, an association between higher autistic traits and delayed menarche was reported in nonclinical female university students. ${ }^{31}$ Ingudomnukul et $\mathrm{al}^{14}$ and Pohl et $\mathrm{al}^{15}$ have demonstrated the relationship between autism and dysmenorrhea in clinically diagnosed ASD samples. The current study found a similar association in typically developing females.

Two prior findings, association between early AAM and $\mathrm{PD}^{1,2}$ and positive correlation between autistic traits and $\mathrm{AAM},{ }^{31}$ would suggest finding lower autistic traits in women with PD, contrary to the results of this study. In our sample, AAM was lower in women with PD; however, this difference was not significant. The mean menarcheal age was 13.01 in the $C G$, which is in line with previous studies conducted in Turkey. ${ }^{32}$ We also did not find any correlations between autistic traits and AAM in this sample. Therefore, the associations between AAM, PD, and ASD should be further investigated.

Previous studies have demonstrated sensory processing (eg, vision, hearing, touch, smell, proprioception) problems in individuals with ASD. ${ }^{33}$ Sensory disturbances in autism may present as hypersensitivity (occurring as there is an overload of stimuli) and hyposensitivity (occurring as an underreaction to the sensory stimuli). ${ }^{34}$ Tavassoli et $\mathrm{al}^{35}$ have reported that adults with ASD had more sensory overresponsivity to stimuli in the environment and within their body. Robertson and Simmons ${ }^{36}$ have demonstrated strong link between autistic traits and sensory processing difficulties in the general population. Our finding, positive correlation between autistic traits and menstrual pain intensity, is in line with the existing literature.

In the current study, subjects with PD reported significantly higher AQ - Total scores than controls and scored significantly higher on only one of the five subscales of AQ, Attention Switching. This subscale of the AQ covers behaviors such as having strong interests, an adherence to routine, and anxiety surrounding new situations. These findings may suggest that PD patients experience difficulties similar to those with ASDs, at least in Attention Switching domain measured by the AQ. Attention Switching subscale might be indicative of a relationship between restricted interests and desire for sameness, and painful menstruation.

One other finding of the current study was association between menstrual pain intensity, measured by VAS, and Attention Switching subscale of the AQ, meaning that females with PD might have poor attention switching abilities. This is consistent with the study of Keogh et $\mathrm{al}^{37}$ concluding that menstrual-related pain was related with greater attentional interference, including attentional switching. Craner et $\mathrm{al}^{38}$ have also reported that women with premenstrual disorders had lower abilities to shift their attention. The perception of pain depends on attention that is allocated to a stimulus, and directing attention away from that stimulus reduces pain. ${ }^{39,40}$ These findings suggest that Attention Switching might be a vulnerability factor by contributing to more painful menstruation.

Several limitations of this study should be kept in mind. First, although our sample was homogeneous, as consisting of medical students, it does not represent all women and we cannot generalize our findings to the general population. Second, as this was a cross-sectional study, we cannot determine the causal relationships between autistic traits and dysmenorrhea. Prospective, longitudinal studies are required to address this issue. Finally, we did not consider several possible confounding factors, including family history of ASD and PD and history of sexual abuse, that might be expected to have an impact on our findings.

\section{Conclusion}

This is the first investigation reporting an association between autistic traits and PD in "neurotypical" female university students. Although the biological underpinning of this relationship is unclear, these preliminary findings require further exploration.

\section{Acknowledgment}

The authors report that the abstract was presented at the "International Meeting for Autism Research" during May 11-14, 2016, at the Baltimore Convention Center, BaltimoreWashington DC, USA, as a poster presentation.

\section{Disclosure}

The authors have no conflicts of interest in this work. 


\section{References}

1. Ju H, Jones M, Mishra G. The prevalence and risk factors of dysmenorrhea. Epidemiol Rev. 2014;36:104-113.

2. Iacovides S, Avidon I, Baker FC. What we know about primary dysmenorrhea today: a critical review. Hum Reprod Update. 2015;21(6): $762-778$.

3. Ju H, Jones M, Mishra GD. A U-shaped relationship between body mass index and dysmenorrhea: a longitudinal study. PLoS One. 2015; 10(7):e0134187.

4. Gagua T, Tkeshelashvili B, Gagua D, McHedlishvili N. Assessment of anxiety and depression in adolescents with primary dysmenorrhea: a case-control study. J Pediatr Adolesc Gynecol. 2013;26(6):350-354.

5. American Psychiatric Association. Diagnostic and Statistical Manual of Mental Disorders. 5th ed. Arlington, VA: American Psychiatric Publishing; 2013.

6. Developmental Disabilities Monitoring Network Surveillance Year 2010 Principal Investigators, Centers for Disease Control and Prevention (CDC). Prevalence of autism spectrum disorder among children aged 8 years - autism and developmental disabilities monitoring network, 11 sites, United States, 2010. MMWR Surveill Summ. 2014;63(2):1-21.

7. Baron-Cohen S, Lombardo MV, Auyeung B, Ashwin E, Chakrabarti B, Knickmeyer R. Why are autism spectrum conditions more prevalent in males? PLoS Biol. 2011;9(6):e1001081.

8. Hallmayer J, Cleveland S, Torres A, et al. Genetic heritability and shared environmental factors among twin pairs with autism. Arch Gen Psychiatry. 2011;68(11):1095-1102.

9. Knickmeyer RC, Baron-Cohen S. Fetal testosterone and sex differences in typical social development and in autism. J Child Neurol. 2006;21(10):825-845.

10. Manning JT, Baron-Cohen S, Wheelwright S, Sanders G. The 2nd to 4th digit ratio and autism. Dev Med Child Neurol. 2001;43(3):160-164.

11. Caufriez A. Menstrual disorders in adolescence: pathophysiology and treatment. Horm Res. 1991;36(3-4):156-159.

12. Hart R, Sloboda DM, Doherty DA, et al. Circulating maternal testosterone concentrations at 18 weeks of gestation predict circulating levels of antimüllerian hormone in adolescence: a prospective cohort study. Fertil Steril. 2010;94(4):1544-1547.

13. Van Anders SM, Watson NV. Menstrual cycle irregularities are associated with testosterone levels in healthy premenopausal women. $\mathrm{Am} \mathrm{J}$ Hum Biol. 2006;18(6):841-844.

14. Ingudomnukul E, Baron-Cohen S, Wheelwright S, Knickmeyer R. Elevated rates of testosterone-related disorders in women with autism spectrum conditions. Horm Behav. 2007;51(5):597-604.

15. Pohl A, Cassidy S, Auyeung B, Baron-Cohen S. Uncovering steroidopathy in women with autism: a latent class analysis. Mol Autism. 2014; $5: 27$

16. Hamilton A, Marshal MP, Murray PJ. Autism spectrum disorders and menstruation. J Adolesc Health. 2011;49(4):443-445.

17. Kyrkou M. Health issues and quality of life in women with intellectual disability. J Intellect Disabil Res. 2005;49(pt 10):770-772.

18. Obaydi H, Puri BK. Prevalence of premenstrual syndrome in autism: a prospective observer-rated study. J Int Med Res. 2008;36(2):268-272.

19. Constantino JN, Todd RD. Autistic traits in the general population: a twin study. Arch Gen Psychiatry. 2003;60(5):524-530.

20. Baron-Cohen S, Wheelwright S, Skinner R, Martin J, Clubley E. The autism-spectrum quotient (AQ): evidence from Asperger syndrome/ high-functioning autism, males and females, scientists and mathematicians. J Autism Dev Disord. 2001;31(1):5-17.
21. Iidaka T, Miyakoshi M, Harada T, Nakai T. White matter connectivity between superior temporal sulcus and amygdala is associated with autistic trait in healthy humans. Neurosci Lett. 2012;510(2):154-158.

22. Gilani SZ, Tan DW, Russell-Smith SN, et al. Sexually dimorphic facial features vary according to level of autistic-like traits in the general population. J Neurodev Disord. 2015;7(1):14.

23. Zettergren A, Karlsson S, Hovey D, et al. Further investigations of the relation between polymorphisms in sex steroid related genes and autistic-like traits. Psychoneuroendocrinology. 2016;68:1-5.

24. Lundström $S$, Chang Z, Råstam $M$, et al. Autism spectrum disorders and autistic like traits: similar etiology in the extreme end and the normal variation. Arch Gen Psychiatry. 2012;69(1):46-52.

25. Collins SL, Moore RA, McQuay HJ. The visual analogue pain intensity scale: what is moderate pain in millimetres? Pain. 1997;72(1-2): 95-97.

26. Köse S, Bora E, Erermis S, Aydın C. Psychometric features of Turkish version of autism-spectrum quotient. Anadolu Psikiyatri Derg. 2010; 11:253-260. (in Turkish).

27. Derogatis LR. The Brief Symptom Inventory (BSI), Administration, Scoring and Procedures Manual. II. Clinical Psychometric Research Inc, Baltimore; 1992.

28. Sahin NH, Durak Batigün A, Uğurtaş S. The validity, reliability and factor structure of the brief symptom inventory (BSI). Turk Psikiyatri Derg. 2002;13(2):125-135. (in Turkish).

29. Fernandes AR, de Sá Rosa e Silva AC, Romão GS, Pata MC, dos Reis RM. Insulin resistance in adolescents with menstrual irregularities. J Pediatr Adolesc Gynecol. 2005;18(4):269-274.

30. Hergüner S, Harmanci H, Hergüner A, Toy H. Autistic traits in women with polycystic ovarian syndrome. Res Autism Spectr Disord. 2012;6(3):1019-1022.

31. Hergüner $S$, Hergüner A. Association between age at menarche and autistic traits in Turkish university students. Am J Hum Biol. 2016; 28(1):44-47.

32. Esen I, Oğuz B, Serin HM. Menstrual characteristics of pubertal girls: a questionnaire-based study in Turkey. J Clin Res Pediatr Endocrinol. 2016;8(2):192-196.

33. Leekam SR, Nieto C, Libby SJ, Wing L, Gould J. Describing the sensory abnormalities of children and adults with autism. J Autism Dev Disord. 2007;37(5):894-910.

34. Horder J, Wilson CE, Mendez MA, Murphy DG. Autistic traits and abnormal sensory experiences in adults. J Autism Dev Disord. 2014;44(6):1461-1469.

35. Tavassoli T, Miller LJ, Schoen SA, Nielsen DM, Baron-Cohen S. Sensory over-responsivity in adults with autism spectrum conditions. Autism. 2014;18(4):428-432.

36. Robertson AE, Simmons DR. The relationship between sensory sensitivity and autistic traits in the general population. J Autism Dev Disord. 2013;43(4):775-784.

37. Keogh E, Cavill R, Moore DJ, Eccleston C. The effects of menstrualrelated pain on attentional interference. Pain. 2014;155(4):821-827.

38. Craner JR, Sigmon ST, Martinson AA. Self-focused attention in response to laboratory stressors among women with premenstrual disorders. Arch Womens Ment Health. 2015;18(4):595-606.

39. Legrain V, Crombez G, Verhoeven K, Mouraux A. The role of working memory in the attentional control of pain. Pain. 2011;152(2): 453-459.

40. Van Damme S, Legrain V, Vogt J, Crombez G. Keeping pain in mind: a motivational account of attention to pain. Neurosci Biobehav Rev. 2010;34(2):204-213. 
Neuropsychiatric Disease and Treatment

Dovepress

\section{Publish your work in this journal}

Neuropsychiatric Disease and Treatment is an international, peerreviewed journal of clinical therapeutics and pharmacology focusing on concise rapid reporting of clinical or pre-clinical studies on a range of neuropsychiatric and neurological disorders. This journal is indexed on PubMed Central, the 'PsycINFO' database and CAS,

and is the official journal of The International Neuropsychiatric Association (INA). The manuscript management system is completely online and includes a very quick and fair peer-review system, which is all easy to use. Visit http://www.dovepress.com/testimonials.php to read real quotes from published authors.

Submit your manuscript here: http://www.dovepress.com/neuropsychiatric-disease-and-treatment-journal 